\title{
PERFORMANCE STUDY OF FOUR EQUALIZATION TECHNIQUES OVER A WIRELESS COMMUNICATION CHANNEL
}

\author{
Anwr ELASYRI ${ }^{*}$, Dogu Cagdas ATILLA ${ }^{2}$, Cagatay AYDIN ${ }^{3}$
}

\begin{abstract}
Equalization techniques are commonly used in low to moderate data transmission over time varying wireless mobile channels. Their use is mainly to alleviate the detrimental effect of intersymbol interference brought about by the multipath phenomena of such channels. The wireless multipath channel is time varying which makes the use of adaptive equalization a necessity to cope with channel variations and consequently to overcome resulting intersymbol interference effects, restore the transmitted symbols, and extract the information being sent. The first part of this paper presents a comparison study of the performances of four representative methods of adaptive equalization. Linear and nonlinear structures each with both LMS and RLS adaptation algorithms are used in the investigation. In the second part of the paper, a computer simulation is carried out to investigate the relationship between the number of propagation paths of the wireless mobile channel and the number of taps in the incorporated adaptive equalizer. Four equalizer structures are used in this investigation which is based on Simulink software package to simulate a communication system incorporating these equalizers. The bit error rate (BER) performance is used as a measure for establishing this dependency.

Key words: intersymbol interference (ISI); LMS and RLS algorithms; linear equalizers, decision feedback equalizers.
\end{abstract}

\section{Introduction}

The IT industries, over the last few decades, have significantly being revolutionized by the invention of internet and the application of the packet switching wireless networks. The former has provided a high speed information transmission via the use of optical fiber webs that virtually covers the whole world. Packet switching wireless networks, on the other hand, have modernized telecommunication systems as they made the dispatch of information possible for the people while they are on the move. The internet technologies have witnessed an extreme development to the extent that made an efficient highspeed data transmission technique over communications channels a necessity of the day. As the rate of the

\footnotetext{
${ }^{1}$ Department of Electrical and Computer Engineering in Graduate School of Science and Engineering, Altinbas University, Istanbul, Turkey, (anwarabdslam@gmail.com) (Dhttps://orcid.org/0000-0001-7570-1782

2 Department of Electrical and Electronics Engineering in School of Engineering and Natural Sciences, Altinbas University, Istanbul, Turkey, (cagdas.atilla@altinbas.edu.tr) Đhttps://orcid.org/ 0000-0002-1895-0333

3 Department of Electrical and Electronics Engineering in School of Engineering and Natural Sciences, Altinbas University, Istanbul, Turkey, (cagatay.aydin@altinbas.edu.tr) (Dhttps://orcid.org/0000-0002-4249-6951

Received: 02 June 2019; Accepted: 24 February 2020

European Journal of Technique (EJT)

Copyright $(\mathcal{C}$ INESEG

(c) (i)

ISSN 2536-5010 | e-ISSN 2536-5134

Doi: https://doi.org/10.36222/ejt.573483

Vol 10, Number 1, 2020 http://dergipark.gov.tr/ejt
} 
data transmission increases to fulfill the needs of the users, the channel introduces distortions in data. One major cause of distortion is Inter Symbol Interference (ISI).

In digital communications, the information is coded and transmitted in the form of rectangular pulses that may assume definite number of levels. The bandwidth of such rectangular pulses is of course infinite. As a pulse propagates through a band limited communication channel, its duration will extend in time with the result of being smeared into adjacent time slots and therefore interfere with the adjacent symbols occupying such slots. This is referred as inter symbol interference (ISI) [1]. Other types of impairments encountered like thermal noise, shot noise, and cross talk will negatively affect the received symbols even further. This makes the use of signal processing techniques at the receiver inevitable in order to overcome the impairments inflicted on the transmitted pulses and consequently facilitate the recovery of their information. Such processing techniques are called "equalization". In principle, the design of a pair of transmitting and receiving filter that can minimize the effect of ISI and the additive noise is feasible provided that the channel characteristics are precisely known [2-5]. However, in general the characteristics of channel are random in the sense that it is one of an ensemble of possible channels [68]. Therefore, using a fixed pair of transmitting and receiving filters will not be adequate if designed on the basis of average channel characteristics as far as ISI is concerned. To overcome this problem adaptive equalization, as shown in Fig. 1, is widely used. The role of equalization is to exercise a precise control so that the channel time response behaves appropriately [3], [9-11].

Adaptive equalizers may be of many different kinds depending on their structure and the adaptation algorithm being implemented. The most commonly used ones are; symbol spaced equalizer, fractionally spaced equalizer, decision-feedback equalizer, and blind equalizer [10].

\section{Related work}

Reference [1] gives good analytical and conceptual information which constitute solid bases for wireless communications in general and networks in particular.

A good overview of the most adaptive techniques is briefly presented in [2], [8], [12]. In [8] FPGA was recommended to replace DSP as more flexibility is provided. Researchers in [3] proposed and investigated an adaptive spatio-temporal equalization for MIMO channels. The proposed solution was tested on a MIMO system and found to give a good adaptation to varying channels resulting in improved performance. The authors in [13] analyzed the use of adaptive filters for equalizing the underwater acoustic channel and compared its behavior when implementing LMS to that when RLS adaptation algorithm is used. In [14] LMS, RLS and PSO (particle swarm optimization) algorithms were used to implement the adaptive channel equalizer. Results measured in terms of mean square error (MSE) and bit error rate (BER) versus number of iterations were obtained. A block minimum mean-squared error equalizer for orthogonal frequency-division multiplexing (OFDM) systems over TV time-varying multipath channels was proposed in [15]. The proposed algorithm used was shown to be less complex and smaller than for other MMSE approaches.

Optimizing the overall impulse response of transmit and receive filters to reduce the frequency selective fading channel to a flat fading one was discussed in [3]. This technique effectively equalizes the received samples as the simulation results had shown. Efficient calculation of the average BER of particular equalization techniques for QPSK transmission over static and quasi-static fading channels was proposed in [9] using an approximate Fourier series method. In [4] a maximum likelihood sequence 
estimation (MLSE) equalizer implemented by the generalized Viterbi algorithm (GVA) was shown to be effective in cancelling both ISI and cochannel interference. A channel-sampling scheme to estimate the channel transitions into and out of the fade state was investigated in [10]. An approach for speeding up the convergence rate and reducing the complexity of equalization was investigated in [5]. This approach is based on combining RLS and LMS algorithms. In [16], a low-complexity per-subcarrier channel equalizer for high-rate wideband transceiver over doubly-dispersive channel was analyzed and its performance was evaluated. Local minima problem during the training mode of most standard adaptive algorithms was addressed in [7], [17] and techniques such as neural network, was recommended to alleviate such limitations. The effect of filter tap-length on the performance of equalized communication system as a function of the number of transmission paths in a multipath environment was tackled in [11].

\section{Rayleigh channel equalization Models}

A multipath, nonstationary communication channel could adequately be represented by a Rayleigh channel model [1], [6], [9]. To alleviate both; the detrimental effects of ISI which arises from the multipath phenomena and the Doppler effects arising from mobility, an adaptive equalizer should be implemented by the receiver as depicted in Fig. 1. Two types of Equalizer structure each with two different adaptation algorithms are investigated in this work.

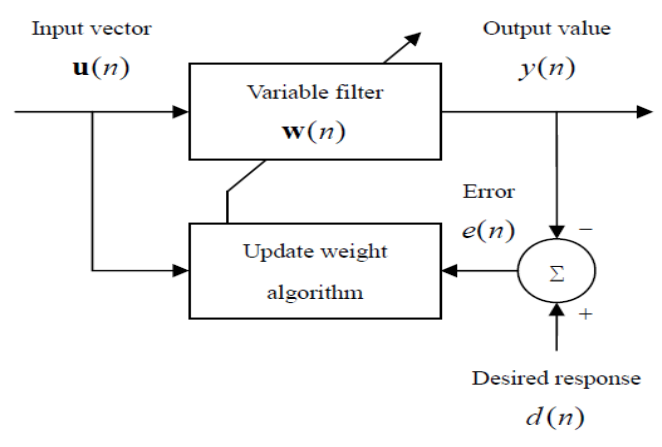

Fig.1. a block diagram of general adaptive filter

\subsection{Linear equalizers}

According to their structures, filters normally distinguish themselves in the context of an adaptive filter with finite memory [2], [8], [11]. A transversal filter is practically implemented using three basic elements, as depicted in Fig. 2: (a) a unit-delay element, (b) multiplication element, and (c) a summing component. The duration of the filter time response is determined by its length, i.e. the number of delay elements used in its structure. Also, the number of delay elements, $\mathrm{M}$ in the figure, defines the filter order. The unit-delay operator $Z^{-1}$ is defined by the unit delay element. The operator, $Z^{-1}$, operates on the input $u(n)$ giving the output $u(n-1)$. The multiplier element in the filter multiplies the tap input by a filter tap weight or filter coefficient.

Thus, a multiplier connected to the $\mathrm{k}^{\text {th }}$ tap input $\mathrm{u}(\mathrm{n}-\mathrm{k})$ produces $\mathrm{W}_{\mathrm{k}} * \mathrm{u}(\mathrm{n}-\mathrm{k})$, where $\mathrm{W}_{\mathrm{k}}$ is the respective tap weight and $\mathrm{k}=0,1, \ldots, \mathrm{M}$. 
The asterisk denotes complex conjugation, which accounts for complex valued tap weights. Individual multiplier outputs are summed producing the overall filter output. For the transversal filter shown, the output is given by

$$
y(n)=\sum_{\mathrm{k}=0}^{\mathrm{M}} \mathrm{w}_{\mathrm{n}}^{*} u(n-k)
$$

The process defined by equation 1 above merely convolves the finite duration impulse response of the filter, $\mathrm{W}_{\mathrm{n}}{ }^{*}$, with the filter input $\mathrm{u}(\mathrm{n})$ to produce the filter output $\mathrm{y}(\mathrm{n})$. This is commonly referred to as a finite convolution.

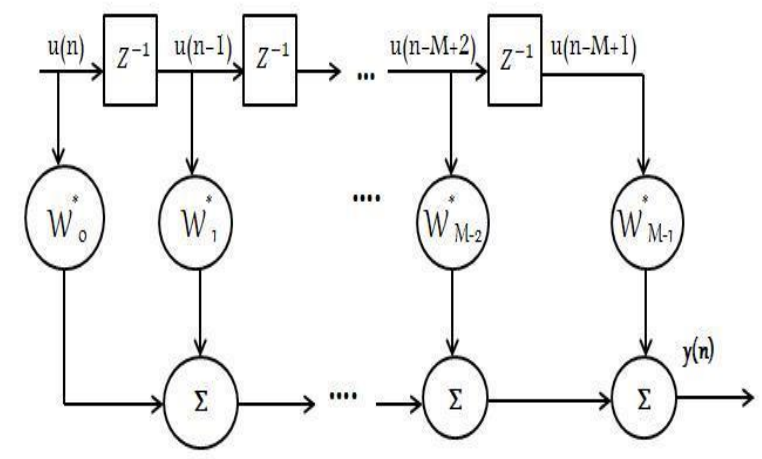

\section{Fig.2. Structural constituent of transversal filter}

\subsection{Feedback Equalizers}

The decision-feedback equalizer (DFE) is the nonlinear adaptive filter. A decision-feedback equalizer consists of two sections; a feedforward section and a feedback section. Both sections are implemented using transversal filters whose taps are spaced at the reciprocal of the symbol rate. The data sequence to be equalized is applied to the input of the feedforward section. Fig. 3 gives an illustration of this type of structure.

Previously detected symbols produce intersymbol interferences. The role of the feedback section is to subtract out the ISI from the estimates of future symbols. This cancellation is an old idea known as the bootstrap technique [14].

A decision-feedback equalizer yields good performance in the presence of severe intersymbol interference, as is experienced in fading radio channels. Fig. 3 shows the block diagram of DFE [3].

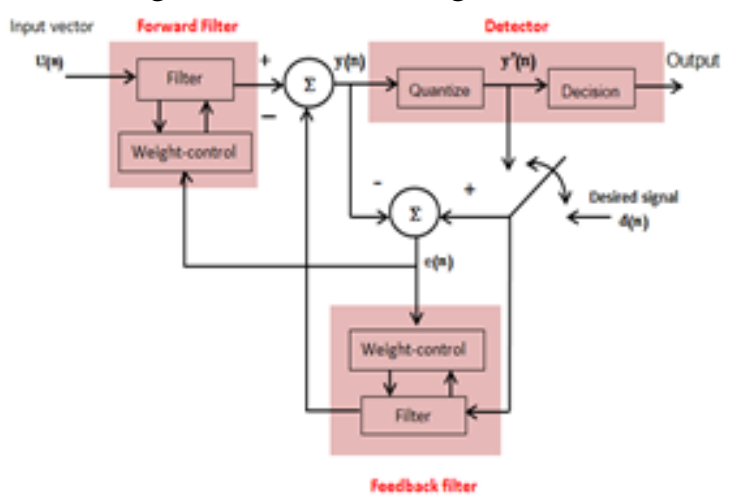

Fig. 3: A functional block diagram of general DFE 


\section{Phase one; Simulation Results and Discussions}

Two types of equalizers were used in this investigation; a linear equalizer and a decision feedback type of equalizer. With each equalizer two kinds of adaptation algorithm, as mentioned previously, were used. The experimental sets up parameters were almost kept the same throughout the experimentation and a table of each is given beside the corresponding result for each experiment. In phase one of experimentations the number of taps is kept fixed and the performance, in terms of bit error rate, is recorded as the energy per bit to noise power ratio is varied. In phase two, the number of propagation paths of the simulated wireless channel is fixed for a given scenario and the number of taps for the equalizer used is varied. The performance in terms of bit error rate is similarly recorded for a range of SNR values. The results obtained will be presented next in the form of graphs. Discussions, explanations and suggestions are given appropriately.

\subsection{Linear Equalizer with LMS Adaptation Algorithm}

Fig. 4 clearly shows that at low to moderate SNR (up to about $12 \mathrm{dBs}$ ) the number of taps of the equalizer has no effect on the performance of the system, but beyond $12 \mathrm{~dB}$ we found that increasing the taps will adversely affect the performance. This is justified on the bases that only three paths were considered in the scenario and at higher SNR increasing the complexity of the equalizer will decrease its rate of convergence and consequently increases the bit error rate.

Table 4.1: Operating parameters for linear equalizer with LMS algorithm

\begin{tabular}{|c|c|c|}
\hline Component & Parameters & Value \\
\hline \multirow{3}{*}{ Variable in Matlab model } & M & 2 \\
\hline & Tsym & 0.0001 \\
\hline & EbNo Vec & $0: 1: 18$ \\
\hline \multirow{3}{*}{ Random Integer Generator } & Set size & $\mathrm{M}$ \\
\hline & Sample time & Tsym \\
\hline & Samples per frame & 1000 \\
\hline \multirow{2}{*}{ BPSK Modulator Base Band } & Phase offset (rad ) & 0 \\
\hline & Output data type & Double \\
\hline \multirow{3}{*}{ Rayleigh Fading } & Maximum Doppler shift & 0.04 \\
\hline & Discrete path delay vector & {$\left[\begin{array}{lll}0, & 0.00000002,0.00000003\end{array}\right]$} \\
\hline & Average path gain Vector & {$[0,-8,-12]$} \\
\hline \multirow[t]{2}{*}{ AWGN Channel } & Number of bits per symbol & $\log 2(\mathrm{M})$ \\
\hline & Symbol period & Tsym \\
\hline \multirow{4}{*}{ Equalizer } & Number of taps $(10,20,30,40)$ & $10,20,30,40$ \\
\hline & Number of samples per symbol & 1 \\
\hline & Reference tap to $(10,20,30,40)$ & $3,6,7,11$ \\
\hline & Step size & 0.0009 \\
\hline \multirow{2}{*}{ BPSK Demodulator Baseband } & Decision type & Hard decision \\
\hline & Phase offset & 0 \\
\hline \multirow{3}{*}{ Error Rate Calculation } & Receive delay to $(10,20,30,40)$ tap & $3,6,7,11$ \\
\hline & Target number of error & 10000 \\
\hline & Maximum number of symbols & 10000000 \\
\hline
\end{tabular}




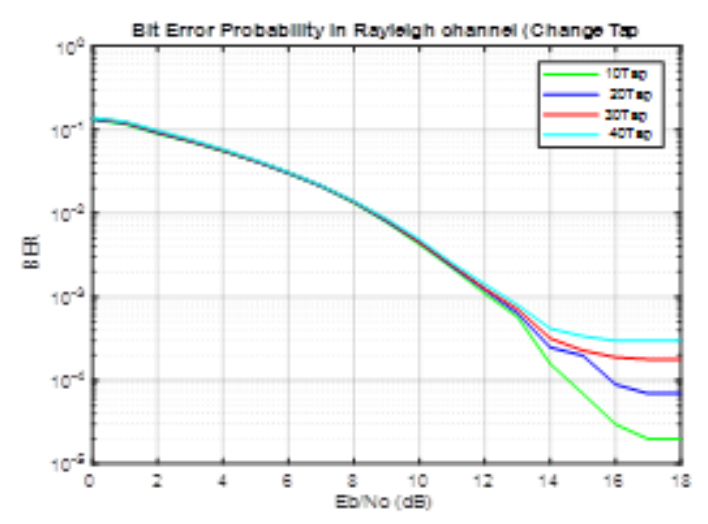

Fig. 4: BER performance for linear equalizer with LMS algorithm

\subsection{Linear equalizer with RLS adaptation algorithm}

For this algorithm the behavior of the system shows a little effect when increasing the equalizer taps. Fig. 5 clearly illustrates the finding already deduced for the linear equalizer with LMS algorithm. At SNRs beyond $14 \mathrm{~dB}$, it is found that higher SNR shows an adverse relationship between the bit error rate and the number of taps of the equalizer as was found with the LMS algorithm and as was deduced earlier. The system implementing linear equalizer with RLS algorithm performs relatively better, in terms of BER than that with LMS algorithm as the graphs in Figs. 4 and 5 indicates.

Table 4.2: Operating parameters for linear equalizer with LMS algorithm (Other parameters are set as in Table 4. 1)

\begin{tabular}{ccc}
\hline Component & Parameters & value \\
\hline \multirow{3}{*}{ Equalizer } & Number of taps $(10,20,30,40)$ & $10,20,30,40$ \\
& Number of samples per symbol & 1 \\
& Reference tap to $(10,20,30,40)$ & $3,6,9,12$ \\
& Forgetting factor & 0.99 \\
\hline
\end{tabular}

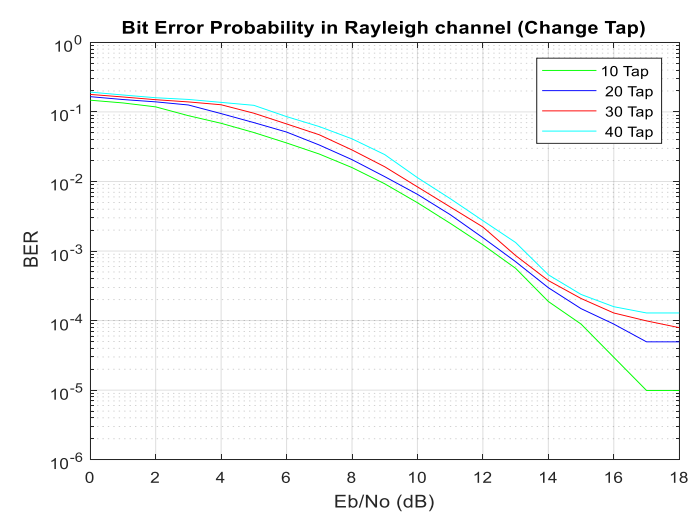

Fig. 5: BER performance for linear equalizer with RLS algorithm

\subsection{DFB equalizer with LMS adaptation algorithm}

The functional components of a general decision feedback equalizer (DFE), is illustrated by the block diagram of Fig. 3. The equalizer in the last experimental set was replaced by a DFE implementing LMS adaptation algorithm. Fig. 6 illustrates the BER rate performance of the decision feedback equalizer for 
four different tap-lengths; 10 taps, 20 taps, 30 taps, and finally 40 taps. The Figure clearly shows that at low SNR (up to about $6 \mathrm{~dB}$ ) the performance of the system is very poor. Over the range 6 to $13 \mathrm{~dB}$, the performance improves significantly. However, over this range varying the tap lengths has no effect as well. Beyond $13 \mathrm{~dB}$, we notice that increasing the tap-lengths will have a negative impact on the system performance, this behavior comes in consistence with the finding already obtained for the linear equalizer.

Table 4.3: Operating parameters for decision feedback equalizer with LMS algorithm (Other parameters are set as in

Table 4.1)

\begin{tabular}{ccc}
\hline Component & Parameters & value \\
\hline \multirow{3}{*}{ Equalizer } & Num of forward tap is $(10,20,30,40)$ & $4,9,14,19$ \\
& Num of feedback tap is $(10,20,30,40)$ & $6,11,16,21$ \\
& Num of samples per symbol & 1 \\
& Reference tap is $(10,20,30,40)$ & $3,6,9,12$ \\
& Step size & 0.0009 \\
\hline
\end{tabular}

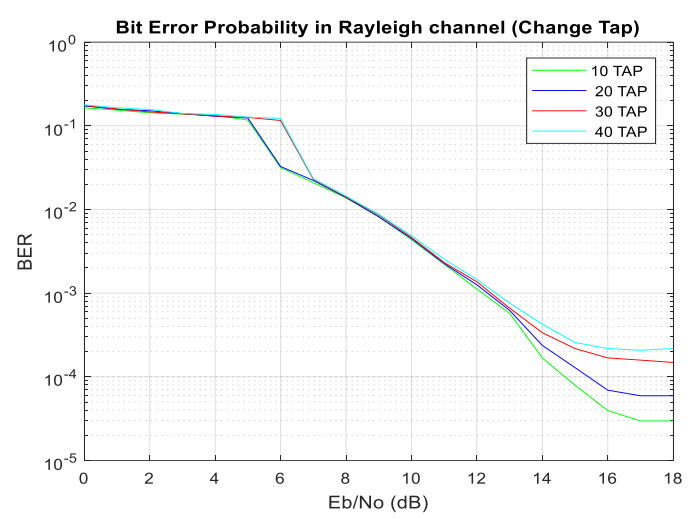

Fig. 6: BER performance for a decision feedback equalizer with LMS algorithm

\subsection{DFB equalizer with RLS adaptation algorithm}

The performance of the system incorporating this type of equalizer that employs RLS adaptation algorithm shows similar behavior to the last system. The graphs in figs. 3, 4, 6 and 7 indicates that about $0.5 \mathrm{~dB}$ is gained by implementing a DFE over the linear equalizer regardless the adaptation algorithm being employed

Table 4.4: Operating parameters for decision feedback equalizer with RLS algorithm (Other parameters are set as in

Table 4.1)

\begin{tabular}{ccc}
\hline Component & parameters & value \\
\hline & Num of forward tap is $(10,20,30,40)$ & $4,9,14,19$ \\
Num of feedback tap is $(10,20,30,40)$ & $6,11,16,21$ \\
Num of samples per symbol & 1 \\
& Reference tap is $(10,20,30,40)$ & $3,6,9,12$ \\
& Forgetting factor & 0.99 \\
\hline
\end{tabular}




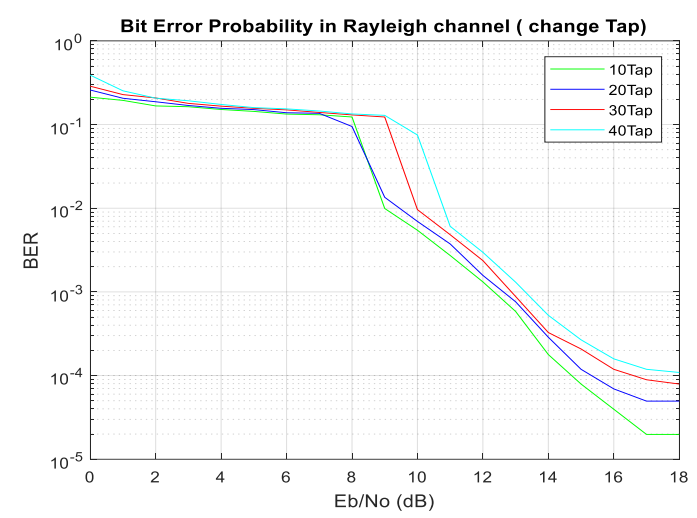

Fig. 7: BER performance for decision feedback equalizer with RLS algorithm

\section{Phase Two; Simulation Results and Discussions}

In this part of investigation, the effect of the number of paths (along which the transmitted signal propagates in the wireless channel) on the performance of a system implementing equalization is investigated. The system is modeled using Simulink software package. The performance is measured in terms of bit error rate (BER). Two different equalizer's structures are used, as in the first part; linear (tapped line) equalizer and decision feedback (nonlinear) equalizer. With each one of them, two adaptation algorithms (the most commonly used ones) are employed. They are; the least mean squares (LMS) algorithm and the recursive least square (RLS) algorithm. The scenario is to have up to seven paths in the channel model with corresponding gains and phase delays. The length of the equalizer (number of taps) is fixed for both structures, and for each particular number of paths; a bit error rate graph is evaluated. The procedure is repeated for the four different models. The results obtained are presented as follow:

\subsection{Linear equalizer with LMS adaptation algorithm}

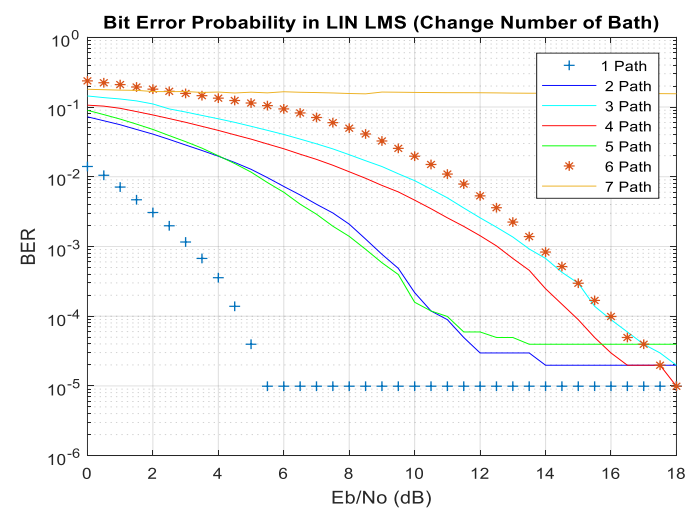

Fig. 8: BER performance for linear equalizer with LMS algorithm 
Table 5.1: Operating parameters for linear equalizer with LMS algorithm

\begin{tabular}{|c|c|c|}
\hline Component & parameters & Value \\
\hline \multirow{3}{*}{ Variable in Matlab model } & M & 2 \\
\hline & Tsym & 0.0001 \\
\hline & EbNoVec & $0: 1: 18$ \\
\hline \multirow{3}{*}{ Random Integer Generator } & Set size & $\mathrm{M}$ \\
\hline & Sample time & Tsym \\
\hline & Samples per frame & 1000 \\
\hline \multirow{2}{*}{ BPSK Modulator Base Band } & Phase offset (rad ) & 0 \\
\hline & Output data type & double \\
\hline \multirow{3}{*}{ Rayleigh Fading } & Maximum Doppler shift & 0.04 \\
\hline & $\begin{array}{l}\text { Discrete path delay vector to number } \\
\text { of Path }(1,2,3,4,5,6,7)\end{array}$ & $\begin{array}{c}{\left[\begin{array}{llll}0 & 0.00000002 & 0.00000003 & 0.00000004 \\
0.00000005 & 0.00000006 & 0.00000007\end{array}\right]}\end{array}$ \\
\hline & $\begin{array}{l}\text { Average path gain Vector to number } \\
\text { of Path }(1,2,3,4,5,6,7)\end{array}$ & {$\left[\begin{array}{lllllll}0 & -6 & -9 & -12 & -13 & -17 & -19\end{array}\right]$} \\
\hline \multirow{2}{*}{ AWGN Channel } & Number of bits per symbol & $\log 2(\mathrm{M})$ \\
\hline & Symbol period & Tsym \\
\hline \multirow{4}{*}{ Equalized } & Number of taps & 5 \\
\hline & Number of samples per symbol & 1 \\
\hline & Reference tap & 2 \\
\hline & Step size & 0.0009 \\
\hline \multirow{2}{*}{ BPSK Demodulator Baseband } & Decision type & Hard decision \\
\hline & Phase offset & 0 \\
\hline \multirow{3}{*}{ Error Rate Calculation } & Receive delay & 2 \\
\hline & Target number of error & 10000 \\
\hline & Maximum number of symbols & 10000000 \\
\hline
\end{tabular}

\subsection{Linear equalizer with RLS adaptation algorithm}

Table 5.2: Operating parameters for linear equalizer with RLS algorithm (Other parameters are set as in Table 5. 1).

\begin{tabular}{ccc}
\hline Component & parameters & value \\
\hline \multirow{2}{*}{ Equalized } & Number of taps & 5 \\
& Number of samples per symbol & 1 \\
& Reference tap & 2 \\
& Forgetting factor & 0.99 \\
\hline
\end{tabular}

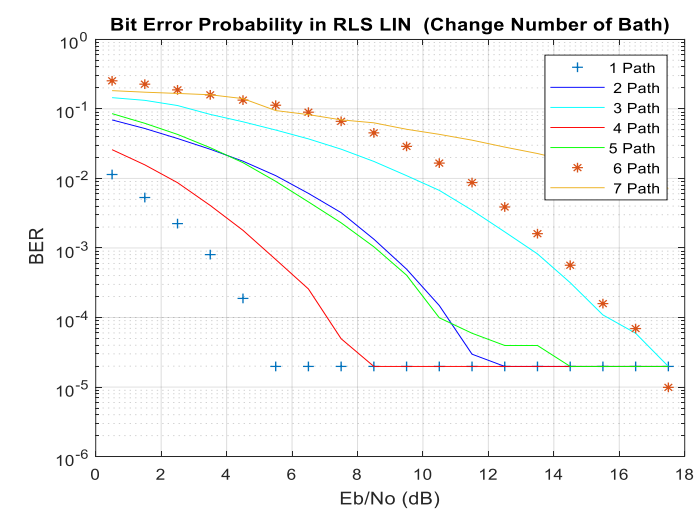

Fig. 9: BER performance for linear equalizer with RLS algorithm 


\subsection{DFB equalizer with LMS adaptation algorithm}

Table 5.3: Operating parameters for DFB equalizer with LMS algorithm (Other parameters are set as in Table 5. 1).

\begin{tabular}{ccc}
\hline Component & parameters & value \\
\hline \multirow{3}{*}{ Equalized } & Number of forward taps & 2 \\
& Number of feedback taps & 3 \\
& Number of samples per symbol & 1 \\
& Reference tap & 2 \\
Step size & 0.0009 \\
\hline
\end{tabular}

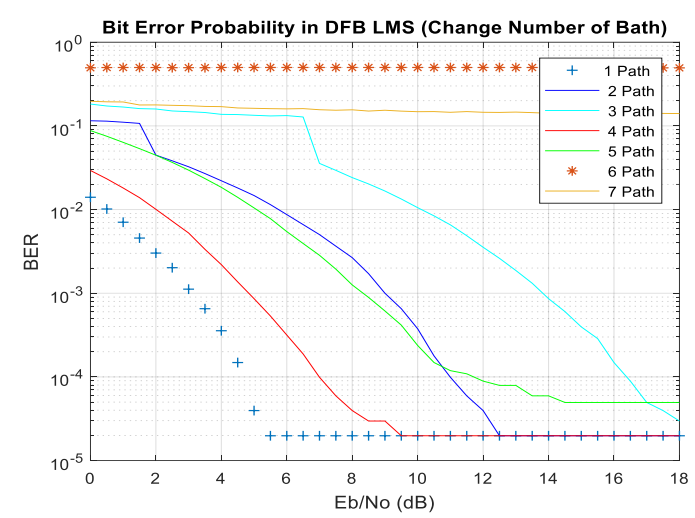

Fig. 10: BER performance for DFB equalizer with LMS algorithm

\subsection{DFB equalizer implementing RLS adaptation algorithm}

Table 5.4: Operating parameters for DFB equalizer with RLS algorithm (Other parameters are set as in Table 5.1).

\begin{tabular}{ccc}
\hline Component & parameters & Value \\
\hline & Number of forward taps & 2 \\
& Number of feedback taps & 3 \\
Equalized & Number of samples per symbol & 1 \\
& Reference tap & 2 \\
& Forgetting factor & 0.99 \\
\hline
\end{tabular}

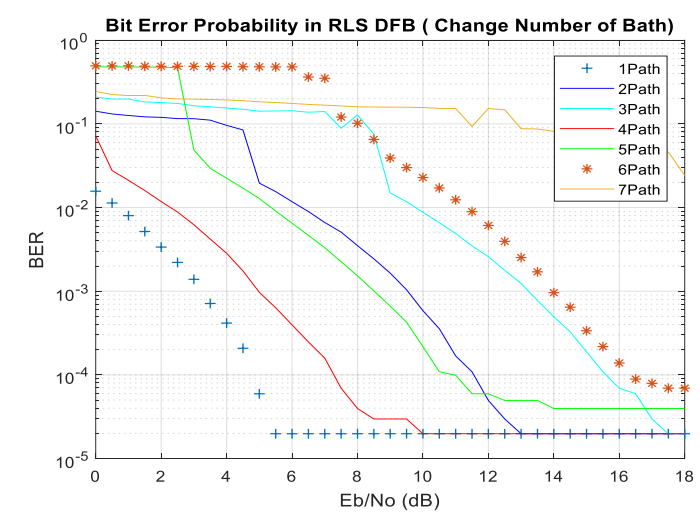

Fig. 11: BER performance for DFB equalizer with RLS algorithm 


\section{CONCLUSION}

The simulation conducted in the first part of this work and the associated results obtained clearly indicate that in such time-varying environment there can be no definite relationship(s) among the structure (complexity or tap-length) of the equalizer, the adaptation algorithm and their impact on the performance of the communication system that uses equalization technique to alleviate the detrimental effect (i.e. ISI) which results due to multipath phenomena. The number of dominant multipath in a particular scenario intuitively recommend a particular number of taps in the implemented equalizer, however the nature of the environment (i.e. being nonstationary) keeps such dominating paths not fixed in number. It is also noted that the linear equalizer with RLS algorithm offers about $0.5 \mathrm{~dB}$ gain over the same equalizer when employs LMS algorithm. Also it noted that the FBE provides about $0.5 \mathrm{~dB}$ advantage over the linear equalizer.

In the second phase of experimentations, the results for the four models show that the best performance is obtained when the number of the multipath (excluding) the main (LOS) path is equal to the number of taps of the equalizer. For a single path, we found best performance for all scenarios. This is intuitive since a single path means that a single copy of the message signal arrives at the receiver and hence no ISI is present. These findings encourage a further investigation in implementing suitable techniques of sensing the channel to establish the number of significant multipath and devising a way of adaptively changing the number of taps in the implemented equalizer. The effect of the reference tap in the transversal structure and the number of proportional taps in the forward and backward in addition to the reference tap in DFB structure are to be determined when varying the tap-lengths of the implemented equalizer.

\section{REFRENCES}

[1] Proakis, J., Salehi, M., Digital Communications, McGraw-Hill Companies, Inc., Fifth Edition, New York, 2008.

[2] Malik, G., Sappal, A. (2011). Adaptive Equalization Algorithms: an Overview. International journal of advanced computer science and applications, vol. 2, no. 3 .

[3] Leon, W., Mengali, U., Taylor, D. (1997). Equalization of Linearly Frequency-Selective Fading Channels. IEEE Transaction on Communication. vol. 45.

[4] Wah Lo, B., Ben Letaief, K. (1999). Adaptive Equalization and Interference Cancellation for Wireless Communication Systems. IEEE Transaction on Communication. vol. 47.

[5] Mosleh, M., AL-Nakkash., A. (2010). Combination of LMS and RLS Adaptive Equalizer for Selective Fading Channel. European Journal of Scientific Research. Vol. 43.

[6] Tehrani, A., Hassibi, B., Cioffi, J. (1999). Adaptive Equalization of Multiple-input Multiple-output (mimo) Frequency Selective Channels. IEEE.

[7] Kundu, D., Nijhawan, G. (2017). Performance Analysis of Adaptive Channel Equalizer Using LMS, Various Architecture of ANN and GA. International Journal of Applied Engineering Research.

[8] Patel, S., Panchal, S., Mewada, H. (2014). Comparative Study of LMS \& RLS Algorithms for Adaptive Filter Design with FPGA. Progress In Science in Engineering Research Journal ( PISER). 
[9] Smee, J., Beaulieu, N., (1998). Error-Rate Evaluation of Linear Equalization and Decision Feedback Equalization with Error Propagation. IEEE Transaction on Communication. vol. 46.

[10] Sharma, P., Chandra, K. Prediction of State Transitions in Rayleigh Fading Channels. IEEE Transaction on Vehicular Technology. vol. 56.

[11] Asim, F., Bashir, S., Salman, M. (2015). Effect of Variation in Filter Length on Adaptive Equalization in Frequency Selective Channels. International Bhurban Conference on Applied Sciences \& Technology (IBCAST), Islamabad, Pakistan.

[12] Khajababu, Md., Padma Sree, Y., Sridha, G V. (2015). Channel Equalization of Adaptive Filters Using LMS and RLS Algorithms. International Journal of Advanced Technology in Engineering and Science, vol. 03.

[13] Dileep, S., Naidu, G B S R., Naveen, V. (2013). Adaptive Channel Equalizer for Wireless Communication System. International journal of electronics and communication engineering (ijece). vol. 2, 159-166.

[14] Sireesha, N., Chithra, K., Tata, S. (2015). Performance Analysis of Linear Recursive Least Squares Adaptive Filter to Mitigate Multipath Effect. Proceedings of Sympol.

[15] Rugini, L., R., Banelli, P., Leus, G. (2005). Simple Equalization of Time-Varying Channels for OFDM. IEEE Communication Letters. vol. 9.

[16] Sharma, P., Chandra, K. Prediction of State Transitions in Rayleigh Fading Channels. IEEE Transaction on Vehicular Technology. vol. 56.

[17] Ihalainen, T., Stitz, T. (2007). Channel Equalization in Filter Bank Based Multicarrier Modulation for Wireless Communications. EURASIP Journal on Advances in Signal Processing.

[18] Khajababu, Md., Padma Sree, Y., Sridha, G V. (2015). Channel Equalization of Adaptive Filters Using LMS and RLS Algorithms. International Journal of Advanced Technology in Engineering and Science, vol. 03.

[19] Dhiman, J., Ahmad, S., Gulia, K. (2013). Comparison between Adaptive filter Algorithms (LMS, NLMS and RLS). International Journal of Science, Engineering and Technology Research (IJSETR) Vol.2. 